\title{
Transforming Cherenkov radiation in metamaterials
}

\author{
Vincent Ginis $^{a}$, Jan Danckaert ${ }^{a}$, Irina Veretennicoff ${ }^{a}$, Philippe Tassin $^{b}$ \\ ${ }^{a}$ Applied Physics Research Group, Vrije Universiteit Brussel, \\ Pleinlaan 2, B-1050 Brussel, Belgium; \\ ${ }^{b}$ Department of Applied Physics, Chalmers University, \\ SE-412 96 Göteborg, Sweden.
}

\begin{abstract}
In this contribution, we explore the generation of light in transformation-optical media. When charged particles move through a transformation-optical material with a speed larger than the phase velocity of light in the medium, Cherenkov light is emitted. We show that the emitted Cherenkov cone can be modified with longitudinal and transverse stretching of the coordinates. Transverse coordinates stretching alters only the dimensions of the cone, whereas longitudinal stretching also changes the apparent velocity of the charged particle. These results demonstrate that the geometric formalism of transformation optics can be used not only for the manipulation of light beam trajectories, but also for controlling the emission of light, here for describing the Cherenkov cone in an arbitrary anisotropic medium. Subsequently, we illustrate this point by designing a radiator for a ring imaging Cherenkov radiator. Cherenkov radiators are used to identify unknown elementary particles by determining their mass from the Cherenkov radiation cone that is emitted as they pass through the detector apparatus. However, at higher particle momentum, the angle of the Cherenkov cone saturates to a value independent of the mass of the generating particle, making it difficult to effectively distinguish between different particles. Using our transformation optics description, we show how the Cherenkov cone and the cut-off can be controlled to yield a radiator medium with enhanced sensitivity for particle identification at higher momentum [Phys. Rev. Lett. 113, $167402(2014)]$.
\end{abstract}

Keywords: Cherenkov radiation, metamaterials, transformation optics, RICH detectors

\section{INTRODUCTION}

Cherenkov radiation ${ }^{1-3}$ is a peculiar form of electromagnetic radiation that arises when charged particles travel through a medium at a velocity greater than the phase velocity of light in that medium. ${ }^{4}$ Experimentally, this radiation was discovered by Pavel Cherenkov ${ }^{5}$ and, theoretically, it was formalized by Ilya Frank and Igor Tamm. ${ }^{6}$ Nowadays, this effect is well understood and has been proven useful in a wide range of applications in applied and experimental physics, ${ }^{7}$ including high-energy particle physics, detection of cosmic rays in astrophysical measurements ${ }^{8}$ development of novel electromagnetic sources,${ }^{9-11}$ localized sensing in biological systems, ${ }^{12}$ spectroscopy of complex nanostructures. ${ }^{13}$

In this contribution, we discuss the generation of Cherenkov radiation in transformation-optical metamaterials. Metamaterials are engineered, man-made materials composed of subwavelength building blocks, densely packed into an effective material. ${ }^{14-16}$ In this way, materials with new optical properties become possible that simply do not exist in either naturally occurring or conventional artificial materials. Metamaterials can be used to realize counterintuitive phenomena like negative refraction ${ }^{17}$ and slow light, ${ }^{18-22}$ and to construct novel devices such as perfect lenses ${ }^{23-25}$ and perfect absorbers. In recent years, there has also been significant interest in the manipulation of Cherenkov radiation inside or in the vicinity of metamaterials. ${ }^{26-37} \mathrm{~A}$ special class of metamaterials is derived from an underlying coordinate transformation. Transformation optics is a framework that exploits the form-invariance of Maxwell's equations in the design of material parameters of optical devices. ${ }^{38,39}$ This design technique has already introduced several new optical devices with unusual properties, such as the invisibility cloak ${ }^{21,40-42}$ and more intricate illusion devices ${ }^{43,44}$ advanced lensing systems, ${ }^{45,46}$

Further author information: (Send correspondence to V.G.)

V.G..: E-mail: vincent.ginis@vub.ac.be

Active Photonic Materials VII, edited by Ganapathi S. Subramania, Stavroula Foteinopoulou, Proc. of SPIE Vol. 9546, 95461Q · @ 2015 SPIE · CCC code: 0277-786X/15/\$18 · doi: 10.1117/12.2190112 
subwavelength cavities, ${ }^{47,48}$ and field manipulators. ${ }^{49,50}$ More recently, the technique has also been extended to more general transformations, ${ }^{51}$ including time-dependent coordinates,${ }^{5-55}$ complex coordinates,${ }^{56}$ and field transformations. ${ }^{57}$

\subsection{Cherenkov Emission in a Transformation-Optical Medium}

In this contribution, we explore the generation of Cherenkov radiation in transformation-optical media-full details of the work can be found in Ref. [47]. We start by considering what happens when a charge moves along the $x$ axis of a transformation-optical medium with material parameters that correspond to a background refractive index $\left(\epsilon_{b}=n_{b}^{2}\right)$ on top of which a linear coordinate stretching along the principle axes has been implemented: $x^{\prime}=f(x), y^{\prime}=g(y), z^{\prime}=h(z)$. Following the equivalence relation of transformation optics, the material parameters of this medium are given by:

$$
\begin{aligned}
& \frac{\epsilon_{x, x}}{\epsilon_{0} \epsilon_{b}}=\frac{\mu_{x, x}}{\mu_{0}}=\frac{g^{\prime}(y) h^{\prime}(z)}{f^{\prime}(x)}, \\
& \frac{\epsilon_{y, y}}{\epsilon_{0} \epsilon_{b}}=\frac{\mu_{y, y}}{\mu_{0}}=\frac{f^{\prime}(x) h^{\prime}(z)}{g^{\prime}(y)}, \\
& \frac{\epsilon_{z, z}}{\epsilon_{0} \epsilon_{b}}=\frac{\mu_{z, z}}{\mu_{0}}=\frac{f^{\prime}(x) g^{\prime}(y)}{h^{\prime}(z)} .
\end{aligned}
$$

The dispersion relation of this medium is gven by

$$
\frac{k_{x}^{2}}{f^{\prime}(x)^{2}}+\frac{k_{y}^{2}}{g^{\prime}(y)^{2}}+\frac{k_{z}^{2}}{h^{\prime}(z)^{2}}=\epsilon_{b} \frac{\omega^{2}}{c^{2}}
$$

We can now apply this dispersion relation to calculate the angle of Cherenkov radiation in a transformationoptical medium. Without loss of generality, we can restrict this analysis to the $x y$ plane. Writing $f^{\prime}(x)=F$, $g^{\prime}(y)=G, h^{\prime}(z)=H$ and defining $\alpha_{\mathrm{PH}}$ as the angle under which the electromagnetic waves are emitted, we find that

$$
\tan \left(\alpha_{\mathrm{PH}}\right)=\frac{k_{y}}{k_{x}}=\frac{G}{F} \frac{\sqrt{F^{2} \epsilon_{b} \omega^{2} / c^{2}-k_{x}^{2}}}{k_{x}}=\frac{G}{F} \tan \left(\alpha^{*}\right),
$$

where $\alpha^{*}$ is the angle of Cherenkov radiation emitted in a medium with refractive index $n_{b} F$. This angle is simply given by the traditional Cherenkov formula and, hence, we find that

$$
\alpha_{\mathrm{PH}}=\arctan \left(\frac{G}{F} \tan \left(\arccos \left(\frac{c}{n_{b} F v}\right)\right)\right) .
$$

The previous result can be better understood from a transformation-optical perspective. In physical space, the charge is moving at velocity $v$ in the $x$ direction. Since this coordinate is stretched by a factor $F$, the particle seems to be moving at velocity $F v$ in the underlying electromagnetic space. In this space, the particle simply travels through an isotropic medium with refractive index $n_{b}$ and, therefore, emits Cherenkov radiation with an opening cone $\theta_{\text {EM }}$ given by

$$
\theta_{\mathrm{EM}}=\arcsin \left(\frac{c}{n_{b} F v}\right) .
$$

Translating the emitted radiation back to the physical space, the $x$ and $y$ components of the cone need to be compressed by a factor $F$ and $G$, respectively, in the underlying electromagnetic space. The angle of the Cherenkov cone then becomes

$$
\theta_{\mathrm{PH}}=\arctan \left(\frac{F}{G} \tan \left(\arcsin \left(\frac{c}{n_{b} F v}\right)\right)\right)
$$




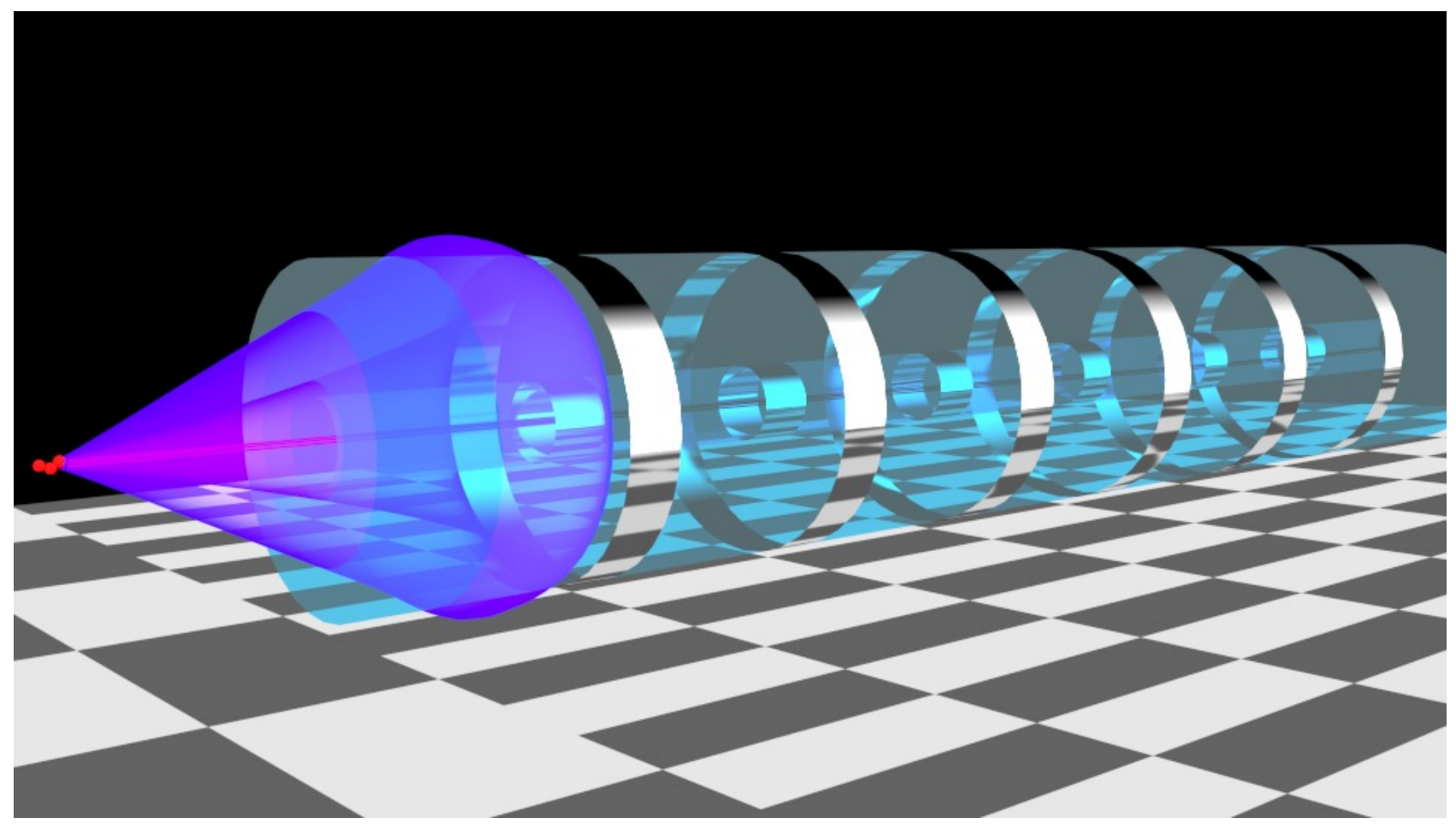

Figure 1. Structure of the metamaterial for modified Cherenkov radiation. The checkerboard pattern indicates the stretching of the underlying coordinate transformation.

\section{DISCUSSION}

It is clear that a transformation of a coordinate in the longitudinal direction has a fundamentally different effect on the Cherenkov radiation than a transformation of a coordinate perpendicular to the direction of propagation. This is related to the fact that a transformation perpendicular to the trajectory of the charged particle only stretches the Cherenkov cone, whereas a transformation along the path of the charge also alters the velocity of the charge in the underlying electromagnetic space. As soon as this velocity drops below the speed of light $c / n_{b}$, Cherenkov radiation ceases to exist. In this respect, it is remarkable how the physics changes before and after a longitudinal coordinate transformation. In contrast to other transformation-optical devices, where existing ray trajectories are manipulated through an ingenious coordinate transformation, we show here that it is possible to start from a system without Cherenkov radiation and transform the nonradiating Liénard-Wiechert potentials into Cherenkov radiation and vice versa. Consider, e.g., a charged particle traveling through a dielectric (with refractive index $n_{b}$ ) at a velocity $v$ smaller than the phase velocity of light in that medium $v<v_{\phi}=c / n_{b}$. Clearly, there is no Cherenkov radiation in this setup. There is a nonradiative electromagnetic field associated to this particle, which is described by the Liénard-Wiechert potentials. Subsequently, it is possible to express this configuration in a novel coordinate system, in which the particle seems to be traveling faster than the speed of light in the dielectric. This is a consequence of a longitudinal transformation that can scale the particle's velocity above or below the Cherenkov cut-off velocity. In this transformed space, there will be emission of Cherenkov radiation. Of course, to implement this transformed space inside a specific material, one needs to change the refractive index of the dielectric $\left(n=n_{b} F\right.$, where $F$ is a scaling factor determined by transformation optics), because of which the phase velocity of light also changes in this medium. In this new medium it is then clear that $v>v_{\phi}=c / n$, which explains the existence of Cherenkov radiation in the transformed medium. In other words, this is an intriguing example where the space before transformation and the space after transformation are fundamentally different. 


\section{ACKNOWLEDGMENTS}

Work at the Vrije Universiteit Brussel was supported by BelSPO (Grant IAP P7-35 photonics@be) and the Research Foundation-Flanders (FWO-Vlaanderen). V. G. is a Postdoctoral Fellow of the Research FoundationFlanders (FWO-Vlaanderen).

\section{REFERENCES}

[1] Cherenkov, P. A., "Radiation of particles moving at a velocity exceeding that of light, and some of the possibilities for their use in experimental physics," Nobel Lectures Physics 1942-1962 , 426-440 (1958).

[2] Frank, I. M., "Optics of light sources moving in refractive media," Nobel Lectures Physics 1942-1962 , $442-468$ (1958).

[3] Tamm, I. E., "General characteristics of radiations emitted by systems moving with super-light velocities with some applications to plasma physics," Nobel Lectures Physics 1942-1962 , 470-482 (1958).

[4] Landau, L. D. and Lifshitz, E. M., [Electrodynamics of Continuous Media], Pergamon, New York (1960).

[5] Cerenkov, P. A., "Visible emission of clean liquids by action of $\gamma$ radiation," Compt. Rend. Acad. Sci. USSR 8, 451 (1934).

[6] Frank, I. M. and Tamm, I. E., "Coherent visible radiation of fast electrons passing through matter," Compt. Rend. Acad. Sci. USSR 14, 109-114 (1937).

[7] Jelley, J. V., [Cerenkov Radiation and its Applications], Pergamon, London (1958).

[8] IceCube Collaboration, "Evidence for high-energy extraterrestrial neutrinos at the IceCube detector," Science 342, 6161 (2013).

[9] Adamo, G., MacDonald, K. F., Fu, Y., Wang, C., Tsai, D., García de Abajo, F. J., and Zheludev, N., "Light well: A tunable free-electron light source on a chip," Phys. Rev. Lett. 103, 113901 (2009).

[10] Liu, S., Zhang, P., Liu, W., Gong, S., Zhong, R., Zhang, Y., and Hu, M., "Surface polariton Cherenkov light radiation source," Phys. Rev. Lett. 109, 153902 (2012).

[11] Vijayraghavan, K., Jiang, Y., Jang, M., Jiang, A., Choutagunta, K., Vizbaras, A., Demmerle, F., Boehm, G., Amann, M. C., and Belkin, M. A., "Broadly tunable terahertz generation in mid-infrared quantum cascade lasers," Nat. Commun. 4, 2021 (2013).

[12] Robertson, R., Germanos, M., Li, C., Mitchell, G., Cherry, S., and Silva, M., "Optical imaging of Cerenkov light generation from positron-emitting radiotracers," Phys. Med. Biol. 54, N355-365 (2009).

[13] García de Abajo, F. J., Pattantyus-Abraham, A., Zabala, N., Rivacoba, A., Wolf, M., and Echenique, P., "Cherenkov effect as a probe of photonic nanostructures," Phys. Rev. Lett. 91, 143902 (2003).

[14] Smith, D. R., Pendry, J. B., and Wiltshire, M. C. K., "Metamaterials and negative refractive index," Science 305, 788-792 (2004).

[15] Soukoulis, C. M. and Wegener, M., "Past achievements and future challenges in the development of threedimensional photonic metamaterials," Nat. Photon. 5, 523-530 (2011).

[16] Liu, Y. and Zhang, X., "Metamaterials: a new frontier of science and technology," Chem. Soc. Rev. 40(5), 2494-507 (2011).

[17] Veselago, V. G., "The electrodynamics of substances with simultaneously negative values of $\epsilon$ and $\mu$," Sov. Phys. Usp. 10, 509-514 (1968).

[18] Zhang, S., Genov, D. a., Wang, Y., Liu, M., and Zhang, X., "Plasmon-Induced Transparency in Metamaterials," Phys. Rev. Lett. 101, 1-4 (2008).

[19] Tassin, P., Zhang, L., Koschny, T., Economou, E. N., and Soukoulis, C. M., "Planar designs for electromagnetically induced transparency in metamaterials," Opt. Express 17, 5595 (2009).

[20] Yannopapas, V., Paspalakis, E., and Vitanov, N., "Electromagnetically induced transparency and slow light in an array of metallic nanoparticles," Phys. Rev. B 80, 1-6 (2009).

[21] Liu, N., Langguth, L., Weiss, T., Kästel, J., Fleischhauer, M., Pfau, T., and Giessen, H., "Plasmonic analogue of electromagnetically induced transparency at the Drude damping limit.," Nat. Mater. 8, 758-62 (2009). 
[22] Verslegers, L., Yu, Z., Ruan, Z., Catrysse, P. B., and Fan, S., "From Electromagnetically Induced Transparency to Superscattering with a Single Structure: A Coupled-Mode Theory for Doubly Resonant Structures," Phys. Rev. Lett. 108, 083902 (2012).

[23] Pendry, J. B., "Negative refraction makes a perfect lens," Phys. Rev. Lett. 85, 3966-3969 (2000).

[24] Tassin, P., Veretennicoff, I., and Van Der Sande, G., "Veselagos lens consisting of left-handed materials with arbitrary index of refraction," Opt. Commun. 264, 130-134 (2006).

[25] Ginis, V., Tassin, P., Soukoulis, C. M., and Veretennicoff, I., "Enhancing optical gradient forces with metamaterials," Phys. Rev. Lett. 110, 057401 (2013).

[26] Luo, C., Ibanescu, M., Johnson, S. G., and Joannopoulos, J., "Cerenkov radiation in photonic crystals," Science 299, 368-371 (2003).

[27] Averkov, Y. O. and Yakovenko, V., "Cherenkov radiation by an electron bunch that moves in a vacuum above a left-handed material," Phys. Rev. B 72, 205110 (2005).

[28] Antipov, S., Spentzouris, L., Gai, W., Conde, M., Franchini, F., Konecny, R., Liu, W., Power, J., Yusof, Z., and Jing, C., "Observation of wakefield generation in left-handed band of metamaterial-loaded waveguide," J. Appl. Phys. 104, 014901 (2008).

[29] Duan, Z., Wu, B.-I., Lu, J., Kong, J. A., and Chen, M., "Cherenkov radiation in anisotropic double-negative metamaterials," Opt. Express 16, 18479-18484 (2008).

[30] Duan, Z., Wu, B.-I., Lu, J., Kong, J. A., and Chen, M., "Reversed Cherenkov radiation in a waveguide filled with anisotropic double-negative metamaterials," J. Appl. Phys. 104, 063303 (2008).

[31] Xi, S., Chen, H., Jiang, T., Ran, L., Huangfu, J., Wu, B.-I., Kong, J. A., and Chen, M., "Experimental verification of reversed Cherenkov radiation in left-handed metamaterial," Phys. Rev. Lett. 103, 194801 (2009).

[32] So, J.-K., Won, J.-H., Sattorov, M., Bak, S.-H., Jang, K.-H., Park, G.-S., Kim, D., and Garcia-Vidal, F., "Cerenkov radiation in metallic metamaterials," Appl. Phys. Lett. 97, 151107 (2010).

[33] Bakunov, M., Mikhaylovskiy, R., Bodrov, S., and Luk'yanchuk, B., "Reversed Cherenkov emission of terahertz waves from an ultrashort laser pulse in a sandwich structure with nonlinear core and left-handed cladding," Opt. Express 18, 1684-1694 (2010).

[34] Duan, Z., Guo, C., and Chen, M., "Enhanced reversed Cherenkov radiation in a waveguide with doublenegative metamaterials," Opt. Express 19, 13825-13830 (2011).

[35] Galyamin, S. N. and Tyukhtin, A. V., "Electromagnetic field of a charge traveling into an anisotropic medium," Phys. Rev. E 84, 056608 (2011).

[36] Vorobev, V. V. and Tyukhtin, A. V., "Nondivergent Cherenkov radiation in a wire metamaterial," Phys. Rev. Lett. 108, 184801 (2012).

[37] Fernandes, D. E., Maslovski, S. I., and Silveirinha, M. G., "Cherenkov emission in a nanowire material," Phys. Rev. B 85, 155107 (2012).

[38] Pendry, J. B., Schurig, D., and Smith, D. R., "Controlling electromagnetic fields," Science 312, 1780-1782 (2006).

[39] Leonhardt, U., "Optical conformal mapping," Science 312, 1777-1780 (2006).

[40] Schurig, D., Pendry, J. B., and Smith, D. R., "Calculation of material properties and ray tracing in transformation media," Opt. Express 14, 9794-9804 (2006).

[41] Li, J. and Pendry, J. B., "Hiding under the carpet: a new strategy for cloaking," Phys. Rev. Lett. 101, 203901 (2008).

[42] Valentine, J., Li, J., Zentgraf, T., Bartal, G., and Zhang, X., "An optical cloak made of dielectrics," Nature Mater. 8, 568-571 (2009).

[43] Lai, Y., Chen, H., Zhang, Z.-Q., and Chan, C. T., "Complementary media invisibility cloak that cloaks objects at a distance outside the cloaking shell," Phys. Rev. Lett. 102(9), 093901 (2009).

[44] Lai, Y., Ng, J., Chen, H., Han, D., Xiao, J., Zhang, Z.-Q., and Chan, C. T., "Illusion optics: The optical transformation of an object into another object," Phys. Rev. Lett. 102, 253902 (Jun 2009).

[45] Leonhardt, U. and Philbin, T. G., "Perfect imaging with positive refraction in three dimensions," Phys. Rev. A. 81, 011804(R) (2010). 
[46] Ma, H. F. and Cui, T. J., "Three-dimensional broadband and broad-angle transformation-optics lens," Nat. Commun. 1, 124 (2010).

[47] Ginis, V., Tassin, P., Soukoulis, C. M., and Veretennicoff, I., "Confining light in deep subwavelength electromagnetic cavities," Phys. Rev. B 82(11), 113102 (2010).

[48] Ginis, V., Tassin, P., Danckaert, J., Soukoulis, C. M., and Veretennicoff, I., "Creating electromagnetic cavities using transformation optics," New J. Phys. 14, 033007 (2012).

[49] Rahm, M., Schurig, D., Roberts, D. A., Cummer, S. A., Smith, D. R., and Pendry, J. B., "Design of electromagnetic cloaks and concentrators using form-invariant coordinate transformations of Maxwell's equations," Photon. Nanostruct.: Fundam. Applic. 6, 87-95 (2008).

[50] Rahm, M., Cummer, S. A., Schurig, D., Pendry, J. B., and Smith, D. R., "Optical design of reflectionless complex media by finite embedded coordinate transformations," Phys. Rev. Lett. 100, 63903 (2008).

[51] Leonhardt, U. and Philbin, T. G., "Transformation optics and the geometry of light," Prog. Optics 53, 69-152 (2009).

[52] Ginis, V., Tassin, P., Craps, B., and Veretennicoff, I., "Frequency converter implementing an optical analogue of the cosmological redshift," Opt. Express 18, 5350-5355 (2010).

[53] Cummer, S. A. and Thomson, R. T., "Frequency conversion by exploiting time in transformation optics," J. Opt. 13, 024007 (2010).

[54] McCall, M. W., Favaro, A., Kinsler, P., and Boardman, A., "A spacetime cloak, or a history editor," J. Opt. 13, 024003 (2011).

[55] Ginis, V., Tassin, P., Koschny, T., and Soukoulis, C. M., "Tunable terahertz frequency comb generation using time-dependent graphene sheets," Physical Review B 91, 161403 (2015).

[56] Castaldi, G., Savoia, S., Galdi, V., Alù, A., and Engheta, N., "PT metamaterials via complex-coordinate transformation optics," Phys. Rev. Lett. 110, 173901 (2013).

[57] Liu, F., Liang, Z., Li, J., and Pendry, J. B., "Manipulating polarization and impedance signature: A reciprocal field transformation approach," Phys. Rev. Lett. 111, 033901 (2013).

[58] Ginis, V., Danckaert, J., Veretennicoff, I., and Tassin, P., "Controlling Cherenkov radiation with transformation-optical metamaterials," Phys. Rev. Lett. 113, 167402 (2014). 